Tropical Journal of Pharmaceutical Research April 2018; 17 (4): 583-588

ISSN: $1596-5996$ (print); 1596-9827 (electronic)

(C) Pharmacotherapy Group, Faculty of Pharmacy, University of Benin, Benin City, 300001 Nigeria.

\title{
Lentinan relieves hepatitis B surface antigen induced functional impairment of monocytes/macrophages
}

\author{
Pei-pei Fang ${ }^{1}$, Jie Li ${ }^{1}$, Shan-shan Huang1, Chen-wei Pan', Guang-yao Zhou', \\ Lingxiang Jin ${ }^{1}$, Lu Zhuge ${ }^{1}$, Le Qin ${ }^{2 *}$ \\ ${ }^{1}$ Department of Infectious Disease, ${ }^{2}$ Department of Pediatric Surgery, The Second Affiliated Hospital and Yuying Children's \\ Hospital of Wenzhou Medical University, 325000, China
}

*For correspondence: Email: Qinle9105@163.com

Sent for review: 15 December 2017

Revised accepted: 26 March 2018

\begin{abstract}
Purpose: To investigate the efficacy of lentinan in relieving hepatitis $B$ surface antigen (HBsAg)induced functional impairment of monocytes/macrophages.

Methods: Monocytic cell line THP-1 was incubated with lentinan and HBsAg for $24 h$ and then

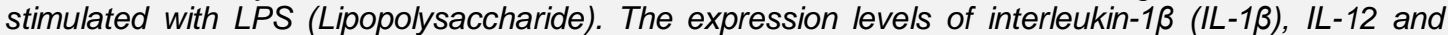
tumour necrosis factor- $\alpha$ (TNF- $\alpha$ ) were measured by enzyme-linked immunosorbent (ELISA) assays and quantitative reverse transcriptase-PCR (q-PCR). Protein levels of IkB- $\alpha$, phospho-ERK, and phospho-p38 were measured by western blotting.

Results: THP-1 cells treated with lentinan and HBsAg showed higher IL-1 $3, I L-12$, and TNF- $\alpha$ levels than cells treated with HBsAg alone. The underlying mechanisms were associated with NF-kB and MAPK signal pathways. Decreased expression of $I k B-\alpha$ and phospho-ERK and increased expression of phospho-JNK and phospho-p38 were observed in cells treated with lentinan and HBsAg when compared with cells treated with HBsAg alone $(p<0.001)$. THP-1 cells incubated with $500 \mu \mathrm{g} / \mathrm{mL}$ lentinan secreted lower levels of cytokines than did control cells after LPS stimulation, suggesting an anti-inflammatory effect for lentinan.

Conclusion: Lentinan shows both pro- and anti-inflammatory functions and may be a promising candidate for hepatitis $B$ virus (HBV) treatment.
\end{abstract}

Keywords: Hepatitis B surface antigen, Lentinan, Immuno-suppression, Pro-inflammatory, Antiinflammatory

\footnotetext{
This is an Open Access article that uses a funding model which does not charge readers or their institutions for access and distributed under the terms of the Creative Commons Attribution License (http://creativecommons.org/licenses/by/4.0) and the Budapest Open Access Initiative (http://www.budapestopenaccessinitiative.org/read), which permit unrestricted use, distribution, and reproduction in any medium, provided the original work is properly credited.

Tropical Journal of Pharmaceutical Research is indexed by Science Citation Index (SciSearch), Scopus, International Pharmaceutical Abstract, Chemical Abstracts, Embase, Index Copernicus, EBSCO, African Index Medicus, JournalSeek, Journal Citation Reports/Science Edition, Directory of Open Access Journals (DOAJ), African Journal Online, Bioline International, Open-J-Gate and Pharmacy Abstracts
}

\section{INTRODUCTION}

Hepatitis B virus (HBV), a non-cytopathic DNA virus with a partially duplex circular genome of $3.2 \mathrm{~kb}$, infects more than 350 million people worldwide $[1,2]$. Chronic HBV infection is the main cause of hepatocellular carcinoma, which is prevalent in the Asian population [3]. Multiple immune-subversion strategies employed by HBV disrupt the host innate and adaptive immune responses and result in systemic immunosuppression [1,4]. Hepatitis B surface antigen 
(HBsAg) is a leading cause of weak antiviral immune responses to HBV infection [5]. High levels of HBsAg are detected in peripheral blood and liver of patients chronically infected with HBV $[6,7]$. Therefore, an important concern with HBsAg is that HBsAg can directly repress the active progression of circulating and liver monocytes/macrophages $[8,9]$. HBsAg or HBV can inhibit the production of type I IFN and IL-12 by antigen presenting cells in a Toll-like receptor (TLR)-dependent manner. Furthermore, the functional activity of monocyte/macrophage were strongly impaired by HBsAg through TLRs, suggesting that HBsAg serves as a suppresser to hamper host innate immune responses $[10,11]$.

Lentinan from shiitake mushrooms (Lentinula edodes) is a $\beta-1,3 \beta$-glucan with $\beta-1,6$ branching and a molecular weight of $500 \mathrm{kDa}$ [12]. Since 1985, lentinan has been approved as an adjuvant for stomach cancer in Japan due to its effects on host immune systems. Lentinan can enhance the functional activity of monocytes/macrophages and the anti-tumour activity of cytotoxic $T$ lymphocytes (CTLs) $[13,14]$. However, the potential of lentinan to treat HBV infection remains unstudied.

In this study, lentinan was used to relieve HBsAg-induced impairment of cytokine secretion by THP-1 cells. The NF-kB and MAPK signalling that was hampered by HBsAg was activated in the presence of lentinan. Lentinan also repressed cytokine production by THP-1 cells upon LPS stimulation. These data indicate that lentinan may be a promising candidate for HBV therapy.

\section{EXPERIMENTAL}

\section{Reagents}

HBsAg was purified from the serum of HBV patients using sucrose gradient centrifugation and affinity chromatography. Lentinan was purchased from Kanghaipharm (Shanghai, China). Polyclonal anti-phospho (p)-ERK, antiERK, and anti-lkB- $\alpha$ and monoclonal antiphospho-JNK, anti-JUK, anti-p-p38, and antip38 were obtained from Cell Signaling Technology (Danvers, MA, USA). Horseradish peroxidise (HRP)-conjugated goat antimouse/rabbit antibodies were obtained from Jackson Laboratory.

\section{Cells and culture conditions}

THP-1 (the human monocytic leukaemia cell line) cells were obtained from ATCC (Manassas,
Virginia, USA) and cultured in complete RPMI 1640 medium which contained additional $10 \%$ FBS, $1 \%$ penicillin $(100 \mathrm{IU} / \mathrm{ml})$, and $1 \%$ streptomycin (100 mg/mL) (Life Technologies, USA) at $37{ }^{\circ} \mathrm{C}$ with $5 \% \mathrm{CO}_{2}$. To activate THP-1 cells for further assays, $50 \mathrm{ng} / \mathrm{ml}$ PMA were first added into the culture media for $24 \mathrm{~h}$. Then with various concentrations of lentinan $(100,250$ and $500 \mu \mathrm{g} / \mathrm{mL}$ ), with or without $25 \mu \mathrm{g} / \mathrm{mL} \mathrm{HBsAg}$ in fresh complete RPMI 1640 were used in the further culture for another $24 \mathrm{~h}$. The cells were washed and stimulated with LPS $(100 \mathrm{ng} / \mathrm{mL})$ for 15 min or 6 h in complete RPMl 1640 medium.

\section{Enzyme-linked immunosorbent assay (ELISA)}

After LPS stimulation for $6 \mathrm{~h}$, secreted IL-1 $\beta$, IL12 , and TNF- $\alpha$ levels were measured by ELISA kits (R \& D Systems, USA) under the guidance of the manufacturer's introduction. Supernatants were added to pre-coated plates in triplicate wells and incubated at $37{ }^{\circ} \mathrm{C}$. After washed, HRP-labelled antibodies were added for $2 \mathrm{~h}$. Substrate was added to washed plates. Chromogenic reactions were stopped with $10 \%$ $\mathrm{H}_{2} \mathrm{SO}_{4}$. Absorbances $450 \mathrm{~nm}$ were measured and the absolute amount of IL-1 $\beta, I L-12$, and TNF- $\alpha$ were calculated based on standard curves.

\section{Total RNA extraction and quantitative reverse-transcriptase-PCR (q-PCR)}

A TRizol reagent (Invitrogen, USA) was used to extract total RNA in THP-1 cells. Then cDNA was synthesized using M-MLV (Promega, USA) under the guidance of the manufacturer's introduction. To assess the gene transcription levels, q-PCR was performed using an ABI Step One Plus Real-Time PCR System (Applied Biosystems, CA, USA) and an EzOmics SYBR qPCR kit (Vazyme Biotech Co., Ltd.) under the guidance of the manufacturer's introduction.

\section{Western blot analysis}

THP- 1 cells were treated with $100 \mathrm{ng} / \mathrm{mL}$ LPS for $15 \mathrm{~min}$. After two times of wash with ice-cold Phosphate Buffer solution (PBS), the cells were lysed in RIPA buffer containing $1 \mathrm{Mm}$ phenylmethanesulfonyl fluoride and centrifuged. The supernatants were collected for protein quantification. Expression levels of IkB-a, p-p38, $\mathrm{p}$-JNK, and p-ERK were analysed. The protein in samples were separated via $12 \%$ SDS-PAGE and transferred to PVDF membranes. Membranes were gently rinsed in deionized water, blocked with skim milk, and incubated 12 $\mathrm{h}$ with the antibodies indicated above (diluted 1: $1000-1: 2000$ in $1 \times$ PBS/0.05\% Tween 20) at 4 ${ }^{\circ} \mathrm{C}$. After washed, Membranes were incubated 
with HRP-conjugated goat anti-rabbit/mouse antibodies for $2 \mathrm{~h}$ at room temperature. Membranes were washed and imaged using ECL detection reagents.

\section{Statistical analysis}

All data were analysed using SPSS 17.0 statistical package and presented as mean \pm standard deviation (SD). ANOVA or Student's ttests were used to determine significance levels; $p<0.05$ was considered significant. The data were processed using GraphPad Prism version 5.

\section{RESULTS}

Lentinan relieves $\mathrm{HBsAg}$-induced functional impairment of THP-1 cells

To investigate if lentinan affects monocytes/macrophages treated with $\mathrm{HBsAg}$, the protein concentrations of IL-1 $\beta, I L-12$, and TNF- $\alpha$ in THP-1 culture supernatants were measured by ELISA (Figure $1 \mathrm{~A}-\mathrm{C}$ ). The results show that HBsAg repressed the expression of these cytokines at the mRNA and protein levels, indicating potent immuno-suppressive activity by lentinan (Figure $1 \mathrm{D}-\mathrm{F}$ ). In the present of $\mathrm{HBsAg}$, addition of lentinan significantly enhanced the production of IL-1 $\beta, \mathrm{IL}-12$, and TNF- $\alpha$ in a dosedependent manner. Interestingly, THP-1 cells incubated with $500 \mu \mathrm{g} / \mathrm{mL}$ lentinan alone showed a mild cytokine response to further stimulation with LPS, suggesting that lentinan induced a certain tolerance of THP-1 cells to LPS that coincided with the multiple functions of the polysaccharide. Thus, in response to further stimulation with LPS, lentinan relieved the functional impairment caused by $\mathrm{HBsAg}$ in monocytes/macrophages.
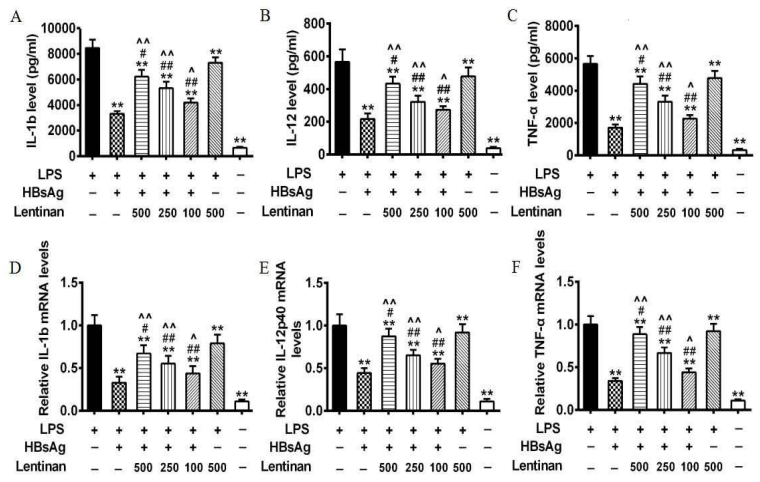

Figure 1: Lentinan relieves HBsAg-induced functional impairment of THP-1 cells. $50 \mathrm{ng} / \mathrm{mL}$ PMA were used to stimulate THP-1 cell differentiation. Stimulated cells were incubated with $\mathrm{HBsAg}(25 \mu \mathrm{g} / \mathrm{mL})$ and/or lentinan $(100,250$, or $500 \mu \mathrm{g} / \mathrm{mL})$ for $24 \mathrm{~h}$. Cells were harvested and stimulated with LPS for another $6 \mathrm{~h}$.
Protein levels of IL-1 $\beta$ (A), IL-12 (B), and TNF- $\alpha(C)$ in culture supernatants were measured by ELISA. RNA was extracted from the cells. Relative IL-1 $\beta$ (D), IL12p40 (E), and TNF- $\alpha$ (F) mRNA levels were measured by q-PCR. Graph are presented as mean and SD. A significance level of $p<0.05, p<0.01$, and $p<0.001$ were labelled with one to three asterisk respectively. ( ${ }^{*}$ vs. control; \# vs. HBsAg; ^ vs. lentinan)

Lentinan restores the function of $\mathrm{HBsAg-}$ treated THP-1 cells by activating NF-kB signalling

Lentinan and HBsAg alter the function of monocytes/macrophages though the NF-kB signalling pathway. Therefore, NF-kB signalling were examined in this cell culture model using western blotting analysis. The engagement of $\mathrm{HBsAg}$ alone inhibited the phosphorylation of IKB- $\alpha$ (Figure 2 A-B). In contrast, addition of lentinan to HBsAg-treated cells promoted the phosphorylation of IKB- $\alpha$, indicating that lentinan induced functional recovery of THP-1 cells at least in part through activation of NF-kB signalling. This result was further confirmed by THP-1 cells treated with lentinan alone. Expression of IKB- $\alpha$ was negatively associated with the production of IL-1 $\beta, \mathrm{IL}-12$, and TNF- $\alpha$. These results suggest that, at least in part, lentinan protected the function of monocytes/macrophages from the effect of HBsAg through competitive activation of NF-kB signalling.

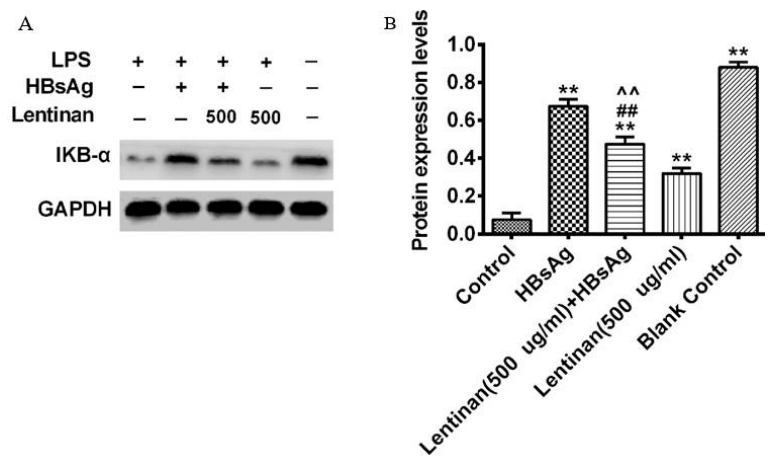

Figure 2: Lentinan reduces $\mid \mathrm{kB}-\alpha$ expression to activate NF-kB signalling in THP-1 cells. (A) IkB- $\alpha$ protein expression was measured by western blotting. (B) Densitometric analysis of (A). The relative expression of IkB- $\alpha$ was normalized to GADPH. Data are presented as mean and SD; ${ }^{*} p<0.05,{ }^{*} p<0.01$, and ${ }^{* * *} p<0.001$ compared with control; \# vs. HBsAg; $\wedge$ vs. lentinan)

Lentinan restores the function of HBsAgtreated THP-1 cells by activating the MAPK signalling pathway

To investigate the role of the MAPK pathway in the process of immune-regulation induced by lentinan, activated THP-1 cells were treated with 
HBsAg, lentinan and HBsAg+lentinan respectively, and then incubated with LPS. Reduced $\mathrm{p}$-ERK expression and increased $\mathrm{p}$ JNK and p-p38 expression were observed in the THP-1 cells that simultaneously incubated with $500 \mu \mathrm{g} / \mathrm{ml}$ lentinan and $25 \mu \mathrm{g} / \mathrm{ml} \mathrm{HBsAg}$ when compared with cells treated with HBsAg alone (Figure 3A-B). The presence of HBsAg facilitated phospho-ERK expression, but dramatically repressed the phosphorylation of JNK and p38. Consistently, the addition of lentinan resulted in reduced expression of $p$-ERK, $p$-JNK, and p-p38 in the cells treated with HBsAg.
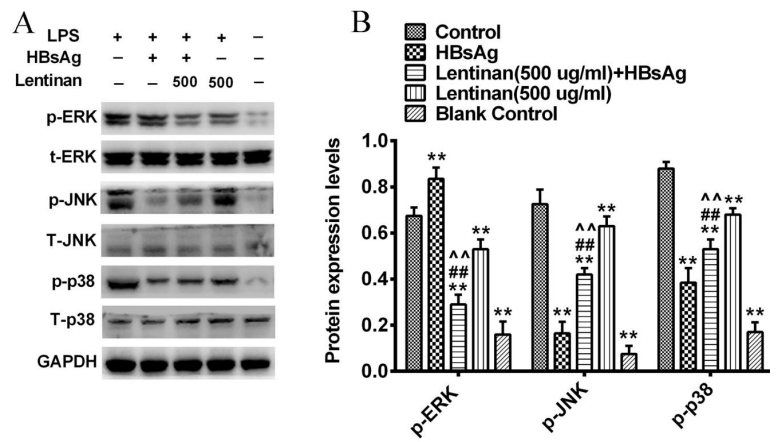

Figure 3: Lentinan regulates the MAPK signalling pathway to relieve $\mathrm{HBsAg}$-induced functional impairment in THP-1 cells. (A) Phospho (p)-ERK, total (T)-ERK, p-JNK, T-JNK, p-p38, and T-p38 protein levels were assessed by western blot. (B) Densitometric analysis of the western blots. Expression levels were normalized to GADPH. Data are presented as mean and $\mathrm{SD} ;{ }^{*} p<0.05,{ }^{* *} p<0.01$, and ${ }^{* * *} p<0.001$ compared with control; \# vs. HBsAg; $\wedge$ vs. lentinan)

\section{DISCUSSION}

Lentinan is an immune response modifier and an anti-cancer drug for stomach cancer [12,14]. Lentinan enhances host immunity by activating monocytes/macrophages and neutrophil antimicrobial functions $[13,15]$. The $\beta$-glucan structure of lentinan can directly bind to dectin-14 that is expressed on the surface of neutrophils, macrophages, and natural killer cells [16]. As a polysaccharide, lentinan also has various effects on inflammatory signalling pathways, such as NF-kB, MAPK, and the inflammasome [17]. Although lentinan promotes the activity of macrophages and CTLs, it may also inhibit the progress of inflammation in vivo by blocking proinflammatory cytokines, such as IL-1 $1 \beta$ and IL-18 [17].

Several studies have examined the immunemodulating effects of lentinan in cancer treatments. Tumour patients who received lentinan enhanced survival rates [14,18]. Administration of lentinan promoted the generation of CTLs, as well as the complement response that helped the CTLs recognize tumour cells. Moreover, macrophage cytotoxic activities and TNF production were enhanced after administration of lentinan [19].

Cancer patients show potent immunosuppression in tumour microenvironments, as well as in the circulatory system [20]. Myeloidderived suppressor cells (MDSCs) induce failure of host anti-tumour immune responses and repress the functions of CTLs, resulting in tumour survival and expansion [21]. Indeed, some MDSCs share the phenotype of a monocyte subset; thus, lentinan may function on these cells to promote host anti-tumour responses. Patients chronically infected with HBV have insufficient antiviral immune responses. These deficits presented as the impairment of myeloid subsets, indicating that the repression of the host innate immune arm may be a leading cause of systematic immuno-suppression [22]. These data suggested that lentinan might have a role in chronic HBV treatment.

In this study, addition of lentinan recovered the production of IL-1 $\beta, \mathrm{IL}-12$, and TNF- $\alpha$ in a dosedependent manner by THP-1 cells that exposed to HBsAg. The underlying mechanisms were associated with activation of NF-kB and MAPK signal pathways. Upon LPS stimulation, THP-1 cells treated with $\mathrm{HBsAg}$ alone showed decreased IkB- $\alpha$ expression; co-incubation with lentinan significantly promoted IkB- $\alpha$ degradation. Thus, lentinan affected NF-kB signalling to recover cytokine production. Since the MAPK signal pathway was indicated in both $\mathrm{HBsAg}$ - and lentinan-induced inflammatory responses, the protein levels of $p$-ERK, p-JNK, and p-p38 protein levels were further measured. THP- 1 cells incubated with lentinan and $\mathrm{HBsAg}$ showed decreased expression of IkB- $\alpha$ and $p$ $E R K$ and increased expression of $p$-JNK and $p$ p38. Notably, the addition of $500 \mu \mathrm{g} / \mathrm{ml}$ lentinan alone inhibited the production of these proinflammatory cytokines, but significantly reduced the expression of IkB- $\alpha$ and repressed process of ERK, JNK, and p38 phosphorylation following LPS stimulation.

In addition to activating the NF-kB and MAPK signalling pathways, lentinan alters inflammasome activation and cytokine maturation [13]. It triggers expression of proinflammatory cytokines via the NLRP3 inflammasome and selectively inhibits cytokine secretion in response to AIM2 inflammasome activation in a colitis model [17]. These findings may explain why lentinan alone decreased cytokine production on further LPS stimulation.

The conventional treatments for HBV infection, including pegylated interferon- $\alpha$ and nucleotide 
analogues, halt the progression of active liver disease, but do not clear HBV infections [23]. These treatments are costly and generally need long-term administration, though they are effective at reducing disease exacerbation. Compared to recombinant interferon- $\alpha$ or nucleotide analogues, lentinan is accessible and shows potential for HBV treatment.

\section{CONCLUSION}

The findings of this study demonstrate that lentinan activates NF-kB and MAPK signalling pathways in vitro to enhance the function of monocytes/macrophages in the presence of HBsAg. These results support the potentials of lentinan in HBV treatment and thus, warrant the need for further studies of its effects in an in vivo model of HPV infection.

\section{DECLARATIONS}

\section{Conflict of Interest}

The authors declare that no conflict of interest is associated with this work.

\section{Contribution of authors}

We declare that this work was done by the authors named in this article and all liabilities pertaining to claims relating to the content of this article will be borne by the authors. Le Qin and Pei-pei Fang designed all the experiments and revised the manuscript. Jie Li, Shan-shan Huang and Chen-wei Pan performed the experiments, and Guang-yao Zhou, Lingxiang Jin and Lu Zhuge wrote the manuscript.

\section{REFERENCES}

1. Wykes MN, Lewin SR. Immune checkpoint blockade in infectious diseases. Nat Rev Immunol 2017.

2. Pan CQ, Duan Z, Dai E, Zhang S, Han G, Wang $Y$, Zhang $H$, Zou $H$, Zhu B, Zhao W, et al. China Study Group for the Mother-to-Child Transmission of Hepatitis, Tenofovir to Prevent Hepatitis B Transmission in Mothers with High Viral Load. N Engl J Med 2016; 374: 2324-2334.

3. Islami F, Miller KD, Siegel RL, Fedewa SA, Ward EM, Jemal $A$. Disparities in liver cancer occurrence in the United States by race/ethnicity and state. CA Cancer J Clin 2017; 67: 273-289.

4. Yang P, Li QJ, Feng Y, Zhang Y, Markowitz GJ, Ning S, Deng $Y$, Zhao J, Jiang $S$, Yuan $Y$, et al. TGF-beta-miR34a-CCL22 signaling-induced Treg cell recruitment promotes venous metastases of HBV-positive hepatocellular carcinoma. Cancer Cell 2012; 22: 291 303.

5. Montineri A, Nigro A, Rosa RL, lacobello C, Larocca $L$, Cappello E, Fiumara PF, Raimondo FD, Fatuzzo F. Treatment induced seroconversion to HBsAb following $H B V$ reactivation in the immunosuppressed haematology and oncology patient: a clinical survey of 5 cases in Catania, Italy. J Clin Virol 2011; 52: 284-287.

6. Samal J, Kandpal M, Vivekanandan P. Molecular mechanisms underlying occult hepatitis $B$ virus infection. Clin Microbiol Rev 2012; 25: 142-163.

7. Miller DJ, Williams AE, Le Bouvier GL, Dwyer JM, Grant $J$, Klatskin G. Hepatitis $B$ in hemodialysis patients: significance of HBeAg. Gastroenterol 1978; 74: 12081213.

8. Wang S, Chen Z, Hu C, Qian F, Cheng Y, Wu M, Shi B, Chen J, Hu Y, Yuan Z. Hepatitis B virus surface antigen selectively inhibits TLR2 ligand-induced IL-12 production in monocytes/macrophages by interfering with JNK activation. J Immunol 2013; 190: 5142-5151.

9. Li H, Zhai N, Wang Z, Song H, Yang Y, Cui A, Li T, Wang G, Niu, Crispe IN, Su L, Tu Z. Regulatory NK cells mediated between immunosuppressive monocytes and dysfunctional T cells in chronic HBV infection. Gut 2017.

10. Vingerhoets J, Michielsen $P$, Vanham G, Bosmans $E$, Paulij W, Ramon A, Pelckmans P, Kestens L, LerouxRoels G. HBV-specific lymphoproliferative and cytokine responses in patients with chronic hepatitis $B$. J Hepatol 1998; 28: 8-16.

11. Hou X, Hao X, Zheng M, Xu C, Wang J, Zhou R, Tian Z. CD205-TLR9-IL-12 axis contributes to CpG-induced oversensitive liver injury in HBsAg transgenic mice by promoting the interaction of NKT cells with Kupffer cells. Cell Mol Immunol 2017; 14: 675-684.

12. Maeda YY, Chihara G. Lentinan, a new immunoaccelerator of cell-mediated responses. Nature 1971; 229: 634

13. Sakamoto $Y$, Watanabe $H$, Nagai $M$, Nakade $K$, Takahashi M, Sato T. Lentinula edodes tlg1 encodes a thaumatin-like protein that is involved in lentinan degradation and fruiting body senescence. Plant Physiol 2006; 141: 793-801.

14. Dennert G, Tucker D. Antitumor polysaccharide lentinan. A $T$ cell adjuvant. J Natl Cancer Inst 1973; 51: 17271729.

15. Nishitani Y, Zhang L, Yoshida M, Azuma T, Kanazawa K, Hashimoto $T$, Mizuno M. Intestinal anti-inflammatory activity of lentinan: influence on IL-8 and TNFR1 expression in intestinal epithelial cells. PLoS One 2013; 8: e62441.

16. Bao H, Sun L, Zhu Y, Ran P, Hu W, Zhu K, Li B, Hou Y, Nie J, Gao $T$, et al. Lentinan produces a robust antidepressant-like effect via enhancing the prefrontal Dectin-1/AMPA receptor signaling pathway. Behav Brain Res 2017; 317: 263-271.

17. Ahn H, Jeon E, Kim JC, Kang SG, Yoon SI, Ko HJ, Kim $\mathrm{PH}$, Lee GS. Lentinan from shiitake selectively attenuates AIM2 and non-canonical inflammasome 
activation while inducing pro-inflammatory cytokine production. Sci Rep 2017; 7: 1314.

18. Wang H, Cai Y, Zheng Y, Bai Q, Xie D, Yu J. Efficacy of biological response modifier lentinan with chemotherapy for advanced cancer: a meta-analysis. Cancer Med 2017; 6: 2222-2233.

19. Shimojoh M, Kojima $T$, Nakajima $K$, Hatta $K$, Katoh $A$, Kurita K. Branched chitins as non-natural immunomodulatory biopolymers: secretions of TNFalpha and NO by direct stimulation of macrophages. Biomacromol 2010; 11: 1212-1216.

20. Parrish JA, Immunosuppression, skin cancer, and ultraviolet $A$ radiation, $N$ Engl $J$ Med 2005, 353: 27122713.
21. Papatriantafyllou M. Tumour immunology: MDSCs come at a cost. Nat Rev Immunol 2011; 11: 440-441.

22. Pallett LJ, Gill US, Quaglia A, Sinclair LV, Jover-Cobos $M$, Schurich A, Singh KP, Thomas N, Das A, Chen A, et al. Metabolic regulation of hepatitis $B$ immunopathology by myeloid-derived suppressor cells. Nat Med 2015; 21 : 591-600.

23. Lucifora J, Xia Y, Reisinger $F$, Zhang $K$, Stadler D, Cheng $X$, Sprinzl MF, Koppensteiner H, Makowska Z, Volz T, et al. Specific and non-hepatotoxic degradation of nuclear hepatitis B virus cccDNA. Sci 2014; 343: 1221-1228. 